\title{
The Impact Of COVID-19 on the Future of the Medical Profession: Reality Versus Expectation for Prospective Medical Students
}

\author{
Jay J Park ${ }^{1 *}$ and Ioannis Georgiou ${ }^{2 *}$ \\ ${ }^{1}$ Edinburgh Medical School, University of Edinburgh, UK \\ ${ }^{2}$ University of Aberdeen, School of Medicine and Dentistry, UK
}

KEYWORDS: Medical education; COVID19; Medical students

\begin{abstract}
MINI-REVIEW
COVID19 has indisputably had a tremendous impact in the medical community. Studies have proved that healthcare professionals are indeed more prone to getting COVID19 [1,2]. It has also been evident for young professionals that mood has been largely impacted during the pandemic [3]. Moreover, this unexpected world-wide outbreak has shed light on previously existing racial inequality in the healthcare system [4]. Despite the recent reports demonstrate that there is an increased amount of medical school applicants [5,6]. As early retirement has become under a spotlight for many doctors, this phenomenon seems to be anti-intuitive and demands interpretation.
\end{abstract}

Both the US and UK displayed three times greater incidences of healthcare professionals contracting the virus compared to those from the public [1]. A UK-based study during the first lockdown denoted a seven-times increase in occurrence of severe COVID19 cases when compared to non-essential workers [2]. This discrepancy in COVID19 incidences between the public and the healthcare workers are reflected in our study, demonstrating the reasons for not participating in the frontline as medical students. The lead cause for not working in the NHS was the predicament of "passing infections to family members" (27\%, $n=606 / 2245)$ followed up by "lack of opportunities" $(22 \%, n=486 / 2245)$, and "risk of getting the infection" (13.9\%, $n=313 / 2245)$ [4].
Our study displayed a decline in mood of medical students and interim foundation (FiY1) doctors due to the pandemic. It is important to note that negative influences on the mood was mainly caused by a disruption in training, such as cancellation of electives and decrease in research opportunities. FiY1 also commented on the toil regarding the need of constantly wearing Personal Protective Equipment's (PPE) at work [3].

The crisis in the workforce during the pandemic has also highlighted the pre-existing issue of racial inequality in the NHS work setting [4]. Our study showed apparent discrepancies between the experiences of BAME and White background young professionals. Regarding information of PPE, only 33\% of the Black, Asian and minority ethnic (BAME) respondents expressed that it was sufficient, as opposed to their White counterpart reporting around $47 \%$. For Infection Prevention and Control (IPC) training, $49 \%$ of the ethnic minority responded that it was adequate while $61 \%$ of the white colleagues expressed the equivalent [7]. $53 \%$ of the BAME professionals did not join the NHS working force due to fear of passing COVID19 to their family and 40\% due to the fear of getting the infection. Regarding the White colleagues only $40 \%$ and $16 \%$ reported the same reason for not working.

Evidence suggests the pandemic has brought forth early retirement of doctors. 2020's Survey of America's Physicians
Quick Response Code:

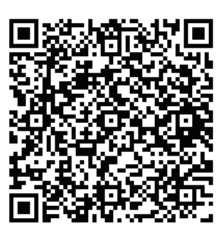

Address for correspondence: Jay J Park, Edinburgh Medical School, University of Edinburgh, UK

Ioannis Georgiou, University of Aberdeen, School of Medicine and Dentistry, UK

Received: May 21, $2021 \quad$ Published: June 08, 2021

How to cite this article: Jay JP, Ioannis G. The Impact Of COVID-19 on the Future of the Medical Profession: Reality Versus Expectation for Prospective Medical Students. 2021- 3(3) OAJBS. ID.000292. DOI: $10.38125 /$ OAJBS.000292 
reports a frightening number of $55 \%$ of the respondents who have experienced over $26 \%$ of financial loss in 2020. In addition, $55 \%$ expect a reduction of income in the upcoming year, 18\% expect a reduced team of staff, and $4 \%$ needing to close their practice. Moreover, The Association of American Medical Colleges (AAMC) reported a shortage of 139000 doctors by the year 2033, which is a $15 \%$ increase from the 2019 report projecting the shortage for the year 2032 [8]. There have been no preceding studies on the issue of early retirement in the UK probably due to the recall of the retirement medical force. However, a report from The Centre for Health Communication Research (CHCR) reports its concern of staff shortages due to early retirement and change in career paths due to COVID19 [9].

Despite the apparent risk of infection in the frontline, negative influence on mood, discrimination of ethnic minorities, and the poor prospects for the current doctors, both UCAS and AAMC reported a dramatic increase in applications to medical schools, $21 \%$ and $17 \%$ respectively $[5,6]$. We are uncertain that the dramatic increase is solely due to altruistic reasons and other motivations should be explored, such as job security due to increase in demands of healthcare professionals. By summarizing the status of medical students and the prospects of the current healthcare workforce, we highlight the importance of a sense of duty in our field of practice, especially in the times of unfavorable circumstances and unsafe environments. Therefore, we carefully express that a mere increase in medical school acceptance rate could potentially raise issues with lack of training opportunities and a high drop-out rate.

\section{REFERENCES}

1. Nguyen L, Drew D, Graham M, Joshi A, Guo C, et al. (2020) Risk of COVID-19 among front-line health-care workers and the general community: A prospective cohort study. The Lancet Public Health 5(9): e475-e483.

2. Mutambudzi M, Niedwiedz C, Macdonald E, Leyland A, Mair F, et al. (2021) Occupation and risk of severe COVID-19: Prospective cohort study of 120075 UK Biobank participants. 78(5).

3. Bandyopadhyay S, Georgiou I, Baykeens B, Gillespie C, Crespo M, et al. (2020) Medical students' mood adversely affected by COVID-19 pandemic: An interim analysis from the SPICE-19 prospective cohort study of 2075 medical students and interim foundation doctors.

4. Georgiou I, Hounat A, Park JJ, Gillespie C, Bandyopadhyay S, et al. (2020) The factors that influenced medical students' decision to work within the NHS during the COVID-19 pandemic- a National, cross-sectional study. J Occup Environ Med Publish 63(4): 296-301.

5. (2020) Students aim high with university applications for next year. UCAS.

6. Weiner S (2020) Applications to medical school are at an all-time high. What does this mean for applicants and schools? AAMC.

7. Norton EJ, Georgiou I, Fung A, Nazari A, Bandyopadhyay S, et al. (2020) Personal protective equipment and infection prevention and control: a national survey of UK medical students and interim foundation doctors during the COVID-19 pandemic. J Public Health 43(1): 67-75.

8. (2020) Survey of America's Physicians. The Physicians Foundations.

9. Nichols B, Underwood J, Hollings J (2020) The rapidly changing NHS Communication in the age of corona virus. High Wycombe: Center for Health Communication Research. 